\title{
Blood endothelial cells: utility from ambiguity
}

\author{
Robert P. Hebbel
}

Division of Hematology-Oncology-Transplantation, Department of Medicine, University of Minnesota, Minneapolis, Minnesota, USA.

\begin{abstract}
In the mid-1990s, my research group began to devise a method to establish endothelial cell cultures from human peripheral blood, with an ultimate goal of examining interindividual heterogeneity of endothelial biology. The initial work, published in the $J C l$ in 2000 , described the method enabling successful attainment of blood outgrowth endothelial cells (BOEC). Truly endothelial, BOEC are progeny of a transplantable cell that originates in bone marrow, a putative endothelial progenitor. Our subsequent experimental work focused upon practical applications of BOEC: their use for gene therapy, tissue engineering, assessment of mutant gene effect, and discovery of heterogeneity in endothelial biology.
\end{abstract}

BOEC, in contrast, are fully differentiated endothelial cells by every measure: morphology, phenotype, organelle content, response to stimuli, observable behaviors, and gene expression pattern. Obtained by in vitro culture, BOEC are progeny of circulating, marrow-derived, transplantable cells that are putative endothelial progenitor cells (1). The latter are now referred to as endothelial colonyforming cells (ECFC); these reside in the vessel wall endothelium at higher numbers than in blood (4). Yet, enough ECFC circulate in the blood that they are found in the early stage of the same cultures from which BOEC ultimately emerge.

The publication prompting this Hindsight (1) arose from my group's effort, beginning in 1996, to obtain endothelial cells by culturing human peripheral blood. In that year, a chance book encounter nurtured my belief that genetic variation establishes interindividual differences in endothelial cell function that, in turn, can contribute to the phenotypic heterogeneity observed in humans with clinical vascular disease. I will return to how we tested this notion in the concluding paragraphs. First, the journey.

A graduate student, Yi Lin, was persuaded to take on the challenge, and over two years of exploratory, iterative refinements, she developed a tractable method to grow endothelial cells from cultured blood. Our subsequent paper published in the JCI on blood outgrowth endothelial cells (BOEC) (1) and especially a paper from another group on so-called "EPC" (intended for endothelial progenitor cells) (2) launched a burgeoning exploration of regenerative cell therapeutics for vascular genesis and repair based on the concept of using endothelial progenitor cells. Regrettably, the consequent literature includes frequent application of ambiguous or errant terminologies to multiple cell types and mixed cell populations. This has served to blur the identities of several distinct cell types that are still often conflated by incautious authorship. Oddly, it is this unfortunate imprecision that accounts for many citations of our 2000 JCI paper!

Attempting to clarify, I present what I find to be the most cogent interpretation of an emergent paradigm that is still hampered by complexities, inconsistencies, and controversies. I acknowledge that, as a result, the response of those in the field will vary from full agreement to ardent disagreement. Be that as it may, Figure 1 depicts the distinctions between the major relevant cell types found in peripheral blood. Circulating endothelial cells (CEC) are detached from the vessel wall endothelium by injurious pathobiologies. CEC have a very limited proliferative ability but may be useful for reporting the status of the endothelium remaining in situ (3). In contrast, cultures of peripheral blood mononuclear cells yield an outgrowth of cells falling into either endothelial or nonendothelial categories, depending upon specific culture method. Eventually, it became clear that the cells originally referred to as "EPC" (2) are actually of hematopoietic origin, with limited growth potential and an inability to produce endothelial cells $(4,5)$. Yet, in certain environments "EPC" provide critical nutritive support for endothelial cell expansion (see below).

\section{Uniquely useful features of BOEC}

Several features render BOEC attractive for use in applications that require cells from a specific donor. Examples include autologous cell therapy and - per my own original interest - examination of the endothelial biology that accompanies a certain disease type, phenotype or genotype. Accessible from peripheral blood, BOEC growth is robust, yielding up to $1 \times 10^{19}$ cells ( 63 doublings), after which they stop growing but remain viable (1). We, of course, did not keep all cells, as that would have amounted to approximately $1 \times 10^{6} \mathrm{~kg}$ of BOEC! These cells still can be produced using a closed system or if animal serum is replaced by autologous human serum (Nguyen and Hebbel, unpublished observations) or a human platelet lysate. BOEC maintain a stable phenotype, as assessed by surface antigen and gene expression, at least through a $1 \times 10^{9}$-fold expansion, although at that point they are attaining some morphologic heterogeneity. Finally, BOEC grown in vitro tolerate cryopreservation and/or gene transfer and thereafter can be further expanded.

\section{Demonstrated (experimental)} uses of BOEC

It appears that the biological, essential role of ECFC is to generate endothelial cells 


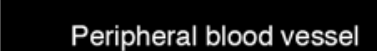

Peripheral blood vessel

Vessel

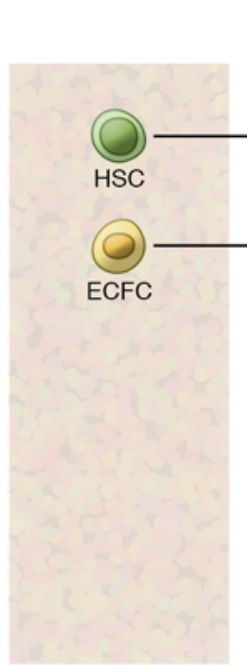

Figure 1. Blood endothelial cell types, origins, and culture. Circulating endothelial cells (CEC) are detached from the vessel wall endothelium as the result of injury and/or disease. Blood outgrowth endothelial cells (BOEC) have all the characteristics of mature endothelial cells. They seem to be progeny of endothelial colony-forming cells (ECFC), a marrow-derived progenitor that resides both in blood and within in situ endothelium. The other relevant cell appearing from appropriate culture of blood mononuclear cells was labeled "EPC" (intended for endothelial progenitor cells). These were later shown to be of hematopoietic stem cell (HSC) origin.

(the biological equivalent of BOEC) when needed. This derives from an emerging paradigm in regenerative medicine that therapeutic promotion of vascular repair/ development probably depends upon a collaboration between ECFC/BOEC and a nutritive source of proangiogenic factors $(6,7)$. Hematopoietic and/or mesenchymal cells that produce these substances likely are provided in contributory therapeutic introductions of "EPC."

In bioengineering, BOEC have been used in the development of bioartificial vascular structures, valve coatings, and nanomatrix materials for wound healing. In cell biology, BOEC cultures enable study of the effect of a given protein on endothelial biology, as has been demonstrated for vWF mutants and endoglin deficiency. Additionally, BOEC have been used to assess epigenetic features of eNOS functional biology and, intriguingly, have been injected as bait and later recaptured to identify the roles of specific signaling molecules during vasculogenesis (8). BOEC also have been used for experimental gene therapy. Using animal models, we evinced the utility of BOEC as carrier vehicles to deliver overexpressed, ther- apeutic proteins: coagulation factor VIII for hemophilia (9); angiostatic proteins for experimental cancer (10); and eNOS for pulmonary hypertension (Somani and Hebbel, unpublished observations). Othfor canine von Willebrand disease.

\section{Diversity of clinical phenotypes}

Returning to the concept that launched our BOEC journey, we did find that surveying BOEC gene expression implicated a specific alteration of endothelial function that conceivably could account for a specific disease phenotype. Predictably, the development of this approach, to enable the survey of genetic influences rather than culture conditions, required technique perfection, adoption of extraordinary precautions, and many standardization and validation experiments $(11,12)$.

First, we studied children with sickle cell anemia (11), all of whom have a systemic inflammatory state; yet, only some develop the inflammatory vasculopathy that causes childhood stroke. The gene expression patterns in BOEC isolated from children with sickle cell anemia suggested that those with vasculopathy possessed vascular ers have used vWF-overexpressing BOEC endothelium that would exhibit exaggerated responsiveness to inflammatory insult. Indeed, when we reestablished cultures of the frozen/stored BOEC from the same children, further study revealed that BOEC from those with vasculopathy exhibited an enhanced NF- $\mathrm{B}$ activation response to TNF/IL-1 stimulation. We do not yet know if the alterations accounting for this are truly genetic or epigenetic. Slightly favoring this outcome being truly genetic is the fact that BOEC themselves have never been exposed to in vivo conditions.

Next, we compared BOEC from African Americans and European Americans (12). Some of the differences we observed in gene expression were consistent with the known disparity in cancer prevalence in these populations, while other variations suggested that the endothelium of the two groups might respond differently to shear stress, a major determinant of endothelial cell homeostasis. As it happens, African Americans tend to have lowered flow-mediated arterial dilation (13), a vasoregulatory response that, indeed, depends upon the signal that flowing blood, via creation of wall shear stress, sends into endothelium. It remains to be seen if testing of individuals will reveal a correlation between such differences in BOEC gene expression and measured variations in shear-stress response. Yet, one wonders if such a difference could contribute to known differences in arterial disease risk among human populations of differing ancestral backgrounds.

With this beginning, we are hopeful that the accessibility and resilience of BOEC will enable continued development of insights that begin to explain the clinical phenotypic heterogeneity that can prevail among humans with any given vascular disease. Although we ourselves chose to exploit the utilitarian opportunities offered by BOEC, it seems that our initial work (1) helped crack open the door, lending just enough light to interest others in seeking an expanded understanding of the fascinating biology of the endothelial cell. We feel privileged to have made a small contribution to these areas of vascular biology.

\section{Acknowledgments}

I am profoundly grateful for the efforts of my laboratory team that conducted these studies and in particular for the 
leadership of Liming Milbauer. The work mentioned herein was funded by: the National Institutes of Health (HL55174, HL62931, DK56326, HL70460, HL71269, HL076540, and HL55552); by Octagen Corporation, COR Therapeutics and Millennium Pharmaceuticals; and by the National Hemophilia Foundation.

Address correspondence to: Robert P. Hebbel, Hematology-Oncology-Transplantation, Department of Medicine, MMC 480, 420 Delaware St. S.E., Minneapolis, Minnesota 55455, USA. Phone: 612.624. 6104; E-mail: hebbe001@umn.edu.

1. Lin Y, Weisdorf DJ, Solovey A, Hebbel RP. Origins of circulating endothelial cells and endothelial outgrowth from blood. J Clin Invest. 2000;105(1):71-77.
2. Asahara T, et al. Isolation of putative progenitor endothelial cells for angiogenesis. Science. 1997;275(5302):964-967.

3. Solovey A, Lin Y, Browne P, Choong S, Wayner E, Hebbel RP. Circulating activated endothelial cells in sickle cell anemia. N Engl J Med. 1997;337(22):1584-1590.

4. Yoder MC, et al. Redefining endothelial progenitor cells via clonal analysis and hematopoietic stem/progenitor cell principals. Blood. 2007;109(5):1801-1809.

5. Medina RJ, et al. Molecular analysis of endothelial progenitor cell (EPC) subtypes reveals two distinct cell populations with different identities. BMC Med Genomics. 2010;3:18.

6. Leeper NJ, Hunter AL, Cooke JP. Stem cell therapy for vascular regeneration: adult, embryonic, and induced pluripotent stem cells. Circulation. 2010;122(5):517-526.

7. Yoder MC. Endothelial progenitor cell: a blood cell by many other names may serve similar functions. JMol Med. 2013;91(3):285-295.

8. Rohban R, et al. Identification of an effective early signaling signature during neo-vasculo- genesis in vivo by ex vivo proteomic profiling. PLoS One. 2013;8(6):e66909.

9. Lin Y, Chang L, Solovey A, Healey JF, Lollar P, Hebbel RP. Use of blood outgrowth endothelial cells for gene therapy for hemophilia A. Blood. 2002;99(2):457-462.

10. Bodempudi V, et al. Blood outgrowth endothelial cell-based systemic delivery of antiangiogenic gene therapy for solid tumors. Cancer Gene Ther. 2010;17(12):855-863.

11. Chang Milbauer L, et al. Genetic endothelial systems biology of sickle stroke risk. Blood. 2008;111(7):3872-3879.

12. Wei P, Milbauer LC, Enenstein J, Nguyen J, Pan W, Hebbel RP. Differential endothelial cell gene expression by African Americans versus Caucasian Americans: a possible contribution to health disparity in vascular disease and cancer. BMC Med. 2011;9:2.

13. Campia U, Choucair WK, Bryant MB, Waclawiw MA, Cardillo C, Panza JA. Reduced endothelium-dependent and -independent dilation of conductance arteries in African Americans. J Am Coll Cardiol. 2002;40(4):754-760. 